\title{
Sur trois contraintes à l'adaptation cinématographique d'À la recherche du temps perdu de Marcel Proust : les cas de Visconti, Pinter, Schlöndorff et Ruiz
}

\author{
Guillaume Lavoie \\ Université Laval
}

How oddly fitting that Harold Pinter, of all people, should have written a screenplay based on Proust's À la recherche du temps perdu. I am not referring now to Pinter's proven ability and considerable experience as a screenwriter nor to the fact that some of his stage plays have been filmed. Rather what seems fitting is that Pinter, a noted practitioner of the Theater of the Absurd, should have undertaken such an apparently absurd task.

Graham, 1982, p. 38 
Ainsi s'exprime Mark Graham à l'égard de l'audacieuse tentative d'Harold Pinter d'adapter À la recherche du temps perdu de Marcel Proust au cinéma. Cette drôle de remarque de Graham illustre bien l'attitude générale qu'ont certains disciples de l'œuvre de Marcel Proust qui pensent qu'il serait absurde, voire impossible, de faire une adaptation cinématographique d'après la Recherche. Il est indéniable que l'œuvre proustienne pose un véritable défi à celui qui veut en faire une adaptation cinématographique. Mais, justement, les difficultés qu'une transposition filmique de la Recherche impose ne devraient pas marquer l'impossibilité d'une telle opération, mais plutôt montrer comment ce travail demande une sérieuse réflexion sur les rapports qu'entretiennent les deux arts impliqués, soit la littérature et le cinéma. En effet, les spécificités de l'œuvre de Proust et sa complexité littéraire exigent de fortes contraintes à sa transposition vers le médium filmique. Loin de représenter une tâche insurmontable, les difficultés inhérentes à l'adaptation filmique de la Recherche constituent, en fait, tout l'intérêt d'une telle entreprise.

Ce que nous proposons dans le présent texte est une analyse de trois des contraintes à l'adaptation cinématographique de la Recherche. À cet effet, nous nous référerons à quatre des six adaptations cinématographiques d'après l'œuvre proustienne réalisées jusqu'à ce jour ${ }^{1}$, soit deux

1 Nous omettons volontairement le film La Captive de Chantal Akerman, réalisé en 2000 , et ce, pour les raisons suivantes. Le film d'Akerman est, si nous pouvons le dire ainsi, une adaptation très libre du texte proustien, et l'une des conséquences de cette liberté est qu'il échappe pour beaucoup aux contraintes d'adaptation que nous désirons aborder. Aussi, La Captive est peut-être moins une adaptation de La Prisonnière qu'un film qui entretient une forte relation intertextuelle avec ce volume de la Recherche. Bref, sans signifier qu'il n'y ait rien à dire sur ce film quant aux contraintes d'adaptation, 
scénarios (qui sont malheureusement demeurés à cette étape préliminaire) ainsi que deux films. Le premier scénario est celui de Luchino Visconti, projet qui avorta en 1972, mais dont le scénario fut publié en 1984. Le second est le scénario d'Harold Pinter, qui aurait été réalisé par le cinéaste américain Joseph Losey et qui fut finalement publié sous le titre The Proust Screenplay en 19782. Le premier film d'après la Recherche projeté sur les écrans de cinéma est signé Volker Schlöndorff et intitulé Un amour de Swann (1984). On devra attendre quinze années avant qu'une autre adaptation voie le jour, qui sera un film de Raoul Ruiz, Le Temps retrouvé, réalisé en 19993. Notre argumentation procédera comme suit: nous exposerons

l'écarter de notre entreprise demeure la solution la plus logique. L'autre adaptation que nous délaissons est un téléfilm de Nina Companéez, originalement diffusé en 2011, simplement intitulé À la recherche du temps perdu. Nous l'écartons de notre travail principalement parce qu'il s'agit d'un projet télévisuel, contrairement aux autres adaptations retenues dans notre corpus. Ayant visionné ledit téléfilm, nous sommes en mesure de juger que son addition au corpus n'ajouterait pas beaucoup de substance à notre propos tel qu'il est déjà formulé. Non pas que le film de Companéez soit sans intérêt, loin de là, mais il faut avouer que l'effort d'adaptation n'est pas très original et qu'à bien des égards, plusieurs choix esthétiques correspondent à une certaine facilité. Cela dit, même si nous nous abstenons de faire mention du téléfilm dans le corps du texte, nous offrirons quelques réflexions en note de bas de page sur le sujet lorsque nous le jugerons opportun.

2 Pour notre travail, nous utiliserons la traduction française du scénario (anglais) d'Harold Pinter qui fut réalisée par les soins de Jean Pavans (2003). Ce choix s'explique principalement parce que l'œuvre proustienne est française ainsi que toutes les autres adaptations, ce qui aura pour effet d'uniformiser la langue des œuvres à l'étude.

3 Il est à noter que des collaborateurs ont participé à la scénarisation de ces adaptations : Joseph Losey et Barbara Bray pour le scénario de Pinter; Suso Cecchi D'Amico au scénario de Visconti; Jean-Claude Carrière, Peter Brook et Marie-Hélène Estienne pour Un amour de Swann de Schlöndorff; Gilles Taurand pour Le Temps retrouvé de Ruiz. Bien que l'effort créateur de ces collaborateurs ne soit pas à négliger, nous ne nous référerons qu'à Pinter, Visconti, Schlöndorff et Ruiz dans le corps du texte par souci de concision. 
chacune des principales contraintes que pose l'œuvre proustienne à sa transposition cinématographique et, pour chaque contrainte, nous analyserons comment chacun des scénarios et films résout, à sa manière, le problème relevé. D'abord nous examinerons comment ces adaptations réagissent devant la longueur démesurée de l'œuvre littéraire à adapter. Ensuite, nous analyserons comment elles traitent la question de la temporalité proustienne au cinéma. Nous terminerons notre réflexion sur les difficultés qu'impose la transposition de la narration proustienne dans une œuvre cinématographique.

\section{La longueur problématique d'À la recherche du temps perdu}

Comment discuter de la difficulté d'adapter cinématographiquement la Recherche sans mentionner d'abord l'immensité de ce roman? Sept tomes en quatre volumes dans son édition de la Pléiade (la plus récente sous la direction de Jean-Yves Tadié), environ 3000 pages en tout, comment réagir devant ce monument romanesque? Il est vrai que la «longueur» des œuvres romanesques est un problème typiquement lié au phénomène d'adaptations cinématographiques d'œuvres littéraires, en ce sens que l'œuvre filmique est contrainte à la durée de projection cinématographique. Bien que la matière expressive du cinéma soit potentiellement plus dense en informations narratives et indicielles que le langage écrit ${ }^{4}$, la nécessité de créer un film dont la durée ne dépasse que

4 Car l'expression audiovisuelle imbrique davantage de «signes» simultanément que la narration littéraire, qui ne peut procéder que mot à 
rarement les quatre heures (ce qui est déjà considéré comme étant très long) conduit souvent au lieu commun que le film contient toujours moins de matière narrative que le roman dont il provient ${ }^{5}$. Cela dit, ce que l'on mentionne moins souvent est le cas inverse, c'est-à-dire que certaines adaptations sont considérées plus longues, ou plus développées narrativement, que le texte littéraire duquel elles s'inspirent 6 . Mais encore, même en avançant l'idée que, dans la majorité des cas, le roman est généralement plus long et détaillé que son adaptation cinématographique, très rares sont les œuvres littéraires qui peuvent rivaliser en longueur avec l'œuvre de Proust, ce qui en fait un cas exceptionnel à ce sujet pour quiconque voulant adapter la Recherche au cinéma. Au-delà de son énormité, c'est aussi la minutieuse structure organique du texte proustien qui devient problématique, car la plupart de ses passages les plus essentiels ne révèlent leur pleine signification qu'une fois mis en parallèle avec d'autres passages de la Recherche. Par

mot, c'est-à-dire un élément à la fois, nonobstant les richesses expressives qui lui sont propres.

5 Alfred Hitchcock croyait d'ailleurs que la longueur du récit filmique correspondait à celui de la nouvelle littéraire : «A film cannot be compared to a play or a novel. It is closer to a short story, which, as a rule, sustains one idea that culmi- nates when the action has reached the highest point of the dramatic curve. As you know, a short story is rarely put down in the middle, and in this sense it resembles a film. And it is because of this peculiarity that there must be a steady development of the plot and the creation of gripping situations which must be presented, above all, with visual skill » (Hitchcock, 1971, p. 72).

6 Mentionnons simplement Brokeback Mountain (2005) d'Ang Lee, dont les 134 minutes de métrage sont définitivement plus longues que la nouvelle éponyme d'Annie Proulx (de son recueil Close Range: Wyoming Stories), qui tient sur une trentaine de pages. Même si la vitesse de lecture est une donnée variable pour chaque lecteur et chaque lecture, on peut statuer que le temps de lecture moyen de la nouvelle est inférieur à la durée de la projection du film et que ce dernier offre une matière narrative plus développée que la nouvelle. 
exemple, l'épisode d'Un amour de Swann n'est véritablement compris qu'une fois que le narrateur lui-même vit un amour excessivement jaloux pour Albertine, et il faut souligner comment plusieurs relations amoureuses décrites dans l'œuvre se juxtaposent elles aussi à cette thématique, comme celles de Charlus et Morel, Robert et Rachel, Basin et Odette, etc. Toutefois, il est inévitable que l'adaptateur doive faire des choix et que, malheureusement, le film ne puisse contenir qu'une infime partie de ce qu'est la Recherche. Examinons désormais comment Pinter, Visconti, Schlöndorff et Ruiz ont procédé dans leur adaptation.

Harold Pinter eut l'audace de créer un scénario qui tente de parcourir la Recherche dans son intégralité. Il explique ainsi sa conception dans la préface de son Scénario Proust:

Durant ma lecture, je pris des centaines de notes, mais restai à la fin très perplexe quant à la façon d'aborder une tâche d'une pareille ampleur. La seule chose dont j'étais certain, c'était que ce serait une erreur de chercher à faire un film centré seulement sur un ou deux volumes, La Prisonnière ou Sodome et Gomorrhe, par exemple. Une adaptation ne serait légitime que si l'on s'efforçait de distiller la totalité de l'œuvre, d'incorporer tous ses thèmes majeurs dans un ensemble compact (p. 11-12).

De ce désir de reprendre la totalité de l'œuvre proustienne, Pinter produit un scénario comptant 207 pages et cumulant hypothétiquement 455 plans, nombre qui aurait probablement augmenté après les étapes cruciales de la réalisation et $\mathrm{du}$ montage, pour une durée d'environ cinq heures. Il est inévitable qu'un film reprenant toute la Recherche ait une durée beaucoup plus longue qu'un film conventionnel, mais à vrai dire, le scénario de Pinter peut bien s'étendre sur trois fois la durée d'un film conventionnel, cela demeure un 
compromis énorme devant l'immensité de la Recherche. Le scénario de Pinter constitue un condensé des éléments narratifs majeurs de la Recherche et il serait faux de prétendre qu'il reprend tous les événements essentiels du roman. Par exemple, la première soirée chez la princesse de Guermantes est absente ainsi que l'épisode à Doncières. Également, des personnages majeurs comme Bloch, Elstir et Bergotte ne font simplement pas partie du scénario. Néanmoins, on doit admettre que la sélection des éléments retenus forme, dans l'ensemble, un résumé efficace du roman proustien ${ }^{7}$, et comme l'avance David Davidson dans son article "Pinter in No Man's land: The Proust Screenplay", cette distillation massive "make the screenplay read like highlights from the novel » (p. 162). Inévitablement, reprendre toute l'histoire de la Recherche pour en faire un seul film oblige une esthétique de la concision et de la brièveté, ce qui n'est pas sans créer un effet de contradiction avec l'esthétique littéraire de la Recherche qui, elle, est démesurément expansive. Mais Pinter est très conscient de cette conséquence et l'assume pleinement, puisqu'il ne cache pas le caractère elliptique et distillé de son scénario par rapport au roman de Proust et décide au contraire d'en faire une loi esthétique de son scénario. À cet égard, Enoch Brater propose que « unlike the architecture of the novel where the narrator

\footnotetext{
7 Il en va de même de l'adaptation de Companéez. Tout lecteur de Proust aura cette drôle d'impression à l'écoute du téléfilm qu'il ne s'agit que d'un résumé, incomplet faut-il le mentionner, de l'œuvre proustienne. En fait, cet aspect paraît plus problématique dans le téléfilm que dans l'adaptation de Pinter, et ce, même s'il est difficile de les comparer sur un même pied puisque le scénario de Pinter n'a jamais été réalisé. Cependant, à la lecture du scénario, on comprend que l'effet résumé est un principe esthétique conscient, que Pinter cherche à en tirer un quelconque profit, tandis que dans le téléfilm de Companéez, l'affaire semble un éternel compromis entre le désir de raconter la Recherche et les contraintes télévisuelles.
} 
builds sustained, stable constructs of memory in generous selections of prose, the screenplay evokes sharp, momentary images encapsulating Proustian duration » (1979, p. 122). Donc, voulant reprendre l'œuvre proustienne dans son intégralité, Pinter a dû composer son scénario par un procédé de condensation extrême de l'œuvre qui, bien que contredisant le style expansif de Proust, s'affiche comme un principe esthétique du film souhaité.

Le scénario de Luchino Visconti, contrairement à celui de Pinter, ne prétend pas tout reprendre de la Recherche pour en faire un film. Mais il le construit à partir d'une très grande partie de la Recherche, qui aurait donné un film d'une longueur comparable à celui de Pinter. Le cinéaste exprime les raisons de sa sélection ainsi :

Mon film sera la peinture d'une société, exactement comme dans Balzac la société de la Restauration. Et en effet ma construction du film commence aux Jeunes filles en fleurs et finit au début de la guerre de 14. Il n'y a même pas Le Temps retrouvé. Il y a ce bloc central qui est la peinture d'une société française de cette époque. (1984, p. 10-11)

En fait, le scénario débute avec l'arrivée de Marcel et de sa grand-mère à Balbec $^{8}$ et inclut une petite partie du Temps

\footnotetext{
8 Le téléfilm de Companéez commence exactement au même moment, soit avec le voyage en train du narrateur et de sa grand-mère en route pour Balbec. Il est possible que Companéez se soit inspirée du scénario de Visconti à ce sujet, car il est étrange que le téléfilm omette entièrement $D u$ côté de chez Swann, tome qui n'est pas moins important que les autres. C'est également ce que Thomas Carrier-Lafleur note dans son livre L'CEil cinématographique de Proust: «On voit donc que cette nouvelle adaptation s'ouvre, [...] comme le scénario de Visconti, par le départ pour Balbec, [...] attestant que Companéez propose à la fois un palimpseste de Proust et de Visconti » (p. 132). Cependant, le choix de Companéez semble grandement justifié par un souci d'économie narrative, car la coupure est assez radicale. Le fait que Visconti avait déjà envisagé une adaptation débutant avec l'arrivée à Balbec a
} 
retrouvé tel qu'il se présente dans la version de la Pléiade, soit le moment où la question de l'écriture est directement posée. À la lecture du scénario, l'argument de Visconti semble très juste : il s'approprie le texte proustien pour faire un film typique de sa cinématographie, une peinture sociale ${ }^{9}$, et motive ainsi son abandon du Côté de chez Swann et de la deuxième partie du Temps retrouvé. Cependant, malgré la cohérence de ces choix, on serait en droit de se demander pourquoi le «Bal de têtes » et «Combray » n'enrichiraient pas la peinture sociale du film de Visconti. Inversement, on peut aussi être surpris que le scénario s'attarde longuement sur le volume de La Prisonnière, qui est une partie plus sentimentale, s'intéressant davantage aux états d'âme du narrateur qu'au monde social décrit dans la Recherche. En fait, les choix scénaristiques de Visconti s'expliqueraient également par un autre principe qu'il énonce de la sorte :

Je ne dois pas faire une transposition littéraire. Évidemment, il $\mathrm{y}$ aura des choses qui se perdront, sûrement une espèce de musicalité proustienne. Mais en échange, je crois pouvoir, avec une image, pénétrer dans cette espèce de labyrinthe profond de Proust, pour vous expliquer un sentiment, une position, une attitude, une tristesse, un moment de jalousie. J'userai de tout ce qui est possible pour rester fidèle au sentiment proustien, pas au style. (ibid., p. 10)

Ce parti pris du sentiment proustien sur le style permet au cinéaste italien de concentrer plus particulièrement son scénario sur les événements dramatiques de la Recherche. En

probablement aidé Companéez à faire ce même choix et ainsi réduire la matière narrative à adapter.

9 Par exemple, son film Il Gattopardo (1963), d'ailleurs adapté du roman du même titre de Giuseppe Tomasi di Lampedusa, peut être considéré comme une peinture de la société aristocratique sicilienne pendant le tumulte de l'unification de l'Italie au début des années 1860. 
outre, bien qu'il montre une structure concise et des effets de brièveté par rapport au texte proustien, ce scénario paraît beaucoup moins condensé que celui de Pinter. Visconti peut développer davantage ses séquences dramatiquement que Pinter et accorde une grande importance à la structure des dialogues, qui sont franchement plus élaborés que ceux de l'auteur anglais, qui mise sur une distillation plus franche. Bref, Visconti opte pour une structure dramatique des événements de la Recherche s'échelonnant sur le bloc central du texte proustien et évite ainsi de faire un film résumé, mais cela a aussi pour conséquence de supprimer plus d'éléments narratifs que le scénario de Pinter.

Le film de Volker Schlöndorff d'après la partie d'Un amour de Swann est certainement la tentative la moins audacieuse d'adaptation de la Recherche qui ait été réalisée jusqu'à présent. Le cinéaste allemand a fait un choix stratégique en reprenant seulement cette partie du roman pour quelques raisons simples. Pascal A. Ifri résume très bien le raisonnement de Schlöndorff dans son article «One Novel, Five Adaptations: Proust on Film » :

[The] idea to concentrate on Un amour de Swann, that is to say one section of the first volume of Proust's novel, makes sense for several reasons: it is relatively short; it tells a complete story; it mainly deals with love and jealousy but it can be viewed as a microcosm of the whole work; and it is the only part of the book not recounted in the first person and thus the easiest to translate to the screen. (p. 23).

Toutes ces raisons expliquent pourquoi Schlöndorff a voulu contourner le problème de la longueur démesurée de la Recherche (et du coup la structure organique qui régit cette œuvre) en considérant la partie d'Un amour de Swann comme 
un récit autonome. Il mise davantage sur un détachement par rapport au texte proustien et peut ainsi forger un film qui donnera l'impression d'être complet en soi, même si les lecteurs de Proust savent très bien que cet épisode, bien qu'aisé à extraire du reste de l'œuvre (comme l'appuie la publication isolée de ce fragment proustien ${ }^{10}$ ), contient une quantité incroyable d'analogies et de parallélismes avec le reste de la Recherche. Schlöndorff prend donc un pari facile en adaptant seulement cette partie et résout le problème de l'immensité de l'œuvre, mais nie un peu du même coup sa forte liaison avec l'intégralité de la Recherche. On pourrait avancer qu'il fait paraître Un amour de Swann plus accessoire qu'il ne l'est en réalité dans la structure globale de l'œuvre proustienne.

Le film de Raoul Ruiz, quant à lui, reprend principalement le dernier tome du texte proustien, Le Temps retrouvé. Ce choix peut sembler étrange, puisque Ruiz décide d'adapter la conclusion de la Recherche, donnant l'impression qu'il manquera des éléments préalables pour comprendre le récit du film. Toutefois, ce choix est beaucoup moins paradoxal qu'il en a l'air, et adapter la Recherche par sa conclusion permet à Ruiz de créer un film qui suit de très près la matière narrative du Temps retrouvé, tout en soulignant constamment la structure organique du texte proustien, puisque Le Temps retrouvé entretient une relation causale très complexe avec le premier tome, Du côté de chez Swann (nous développerons davantage ce propos ultérieurement). Donc, Ruiz peut réaliser un film de 155 minutes qui représente convenablement les événements cruciaux narrés dans Le Temps retrouvé. Cependant, il ne se

10 Entre autres éditeurs, Gallimard propose Un amour de Swann comme roman autonome dès 1930 . 
contente pas du texte proustien et invente un prologue représentant un Marcel Proust fictionnel dans son appartement de la rue Hamelin, qui dicte à sa domestique Céleste Albaret la dernière page écrite par l'auteur réel. Dans L'OEil cinématographique de Proust, Thomas Carrier-Lafleur précise :

Or, ce que dicte Marcel n'est pas à proprement parler un passage de la Recherche. Le film de Ruiz effectue d'emblée un saut qualitatif: il s'agit plutôt de corrections que Proust comptait apporter à la dactylographie de La Prisonnière, au sujet de la mort de Bergotte. Ruiz a repris les phrases exactes que dictait Proust la veille de sa mort, dans la nuit du 17 au 18 novembre 1922, que l'on retrouve dans les notes et variantes de l'édition de la Recherche dans la Bibliothèque de la Pléiade. (p. 135)

En fait, ce prologue consiste vraisemblablement en une adaptation des propos de Céleste Albaret contenus dans une émission de télévision française où cette dernière raconte les derniers moments de l'écrivain ${ }^{11}$. Le cinéaste utilise ce prologue pour placer ce Marcel Proust auteur près de sa mort, ce qui est utile à la fiction de Ruiz, nous y reviendrons. Il ajoute également un épilogue se déroulant sur la plage de Balbec et deux scènes énigmatiques dans une salle ornée de chapeaux haut-de-forme méthodiquement disposés sur le sol, scènes qui ne figurent pas dans la Recherche. Le cinéaste chilien se permet donc de créer un film d'une durée raisonnable qui tente de suivre de très près Le Temps retrouvé, tout en faisant déborder son adaptation des limites du roman en incluant des inventions de son cru, qui ne sont pas aussi farfelues qu'elles paraissent à première vue, ce dont nous discuterons plus bas.

11 Il s'agit en fait de Portrait-souvenir, une émission de Roger Stéphane. L'épisode sur Marcel Proust date de 1962. On peut le visionner dans son intégralité sur le site YouTube. 
Maintenant que nous avons illustré comment ces quatre adaptations cinématographiques de la Recherche tentent de résoudre, chacune à leur manière, le problème de la longueur de l'œuvre, ne serait-il pas possible d'imaginer une solution idéale à ce problème? Évidemment, notre position est purement spéculative, et on pourra reprocher tant qu'on veut à chacune de ces adaptations de ne pas reprendre absolument tout ce qui est narré dans la Recherche, on passerait à côté de l'essentiel puisque ce n'est pas leur but en tant que créations cinématographiques. Mais imaginons un moment qu'il faille trouver le meilleur compromis pour adapter toute la matière narrative de la Recherche. Évidemment, on pourrait penser que la forme de la télésérie permettrait d'étaler sur une durée filmique considérable le roman proustien, faisant miroiter un contexte adéquat pour adapter en long et en large la Recherche ${ }^{12}$. Mais demeurant réaliste, il nous paraît inévitable que les contraintes télévisuelles risquent de causer une multitude de problèmes sur le plan esthétique. Pensons simplement à la rigidité du temps de diffusion. Comment confiner en épisodes de 30 ou 60 minutes la Recherche sans que les codes et stéréotypes les plus convenus de l'esthétique de la télésérie gâchent la liberté et l'originalité discursive du texte proustien? À notre avis, il est préférable qu'une adaptation de

\footnotetext{
12 Il faut mentionner ici que Companéez n'a pas été au bout de cette idée, car son téléfilm dure 230 minutes, ce qui constitue à peine deux fois le métrage conventionnel d'un film. L'œuvre de Companéez souffre ainsi autant de la contrainte du métrage que les autres adaptations, mais en plus, elle est limitée en moyens techniques par le budget modeste et le court temps de réalisation qu'une telle production télévisuelle impose. Cela dit, la facture historique de la production demeure respectable, même si une saveur télé-théâtre reste persistante tout du long. Le grand problème est que le téléfilm est passablement dénué d'originalité quant à la réalisation: on pourrait difficilement filmer la Recherche de façon plus conventionnelle.
} 
l'œuvre proustienne profite d'un traitement s'éloignant autant que possible de toutes contraintes de création et, à cet égard, le mode de production du cinéma d'auteur (profitant d'un financement adéquat) demeure vraisemblablement la meilleure alternative. Ainsi, c'est peut-être Davidson qui énonce le compromis le plus raisonnable, alors qu'il discute du scénario de Pinter : «In wishing to "be true to" Proust's work, might not Pinter have followed the master's example and allowed the rich textures of Remembrance of Things Past to be traced luxuriously throughout a series of films instead of choppily confining them to one? » (1982, p. 158) En effet, une série de films semble une excellente alternative. On peut s'imaginer qu'une quinzaine de films de deux ou trois heures réussirait à intégrer presque tout le récit de la Recherche, ce qui représenterait toutefois une entreprise colossale, mais virtuellement réalisable. Du moins, on peut toujours y rêver...

\section{La complexité de la structure temporelle du roman proustien}

Passons désormais à l'analyse de notre deuxième contrainte à l'adaptation cinématographique de la Recherche, c'est-à-dire sa structure temporelle. Car non seulement la question du temps est essentielle dans ce roman, c'est également à cause de sa structure narrative extrêmement complexe que cette œuvre littéraire est incommode à transcrire en un équivalent cinématographique. Cette difficulté à transposer la temporalité proustienne au cinéma est principalement due à une différence cruciale entre les spécificités expressives des médiums littéraire et cinématographique par rapport au temps. Dans 
Novels into Film, George Bluestone résume cette différence de la sorte : "novel has three tenses; the film has only one » (p. 48). Précisons qu'ici, nous ne parlons pas du temps narratif, mais de l'expressivité temporelle de chacun des médiums. Car on pourrait croire que l'analepse et la prolepse permettent à l'image cinématographique de s'exprimer au passé et au futur, mais ces procédés relèvent de la structure du récit (tout comme c'est le cas en littérature), qui est immanent au médium qui le prend en charge ${ }^{13}$. La littérature utilise le langage comme matériau d'expression, et le langage articule aisément le passé, le présent et le futur par le biais des temps de verbes. Le cinéma utilise l'image en mouvement et le son comme matériaux d'expression, et le caractère perceptuel de ces matériaux confine le cinéma à toujours s'exprimer au présent. L'image montre des événements qui se déroulent comme s'ils prenaient place dans le présent le plus immédiat. Cette différence expressive entre la littérature et le cinéma est particulièrement problématique quant à une adaptation de la Recherche, puisque le roman proustien articule de manière très sophistiquée l'expression temporelle spécifique au langage. Les éléments narrés au présent sont souvent mis en perspective avec des événements passés, et même parfois selon un mode prospectif (hypothétique ou factuel) de péripéties à venir. Cette complexité temporelle découle pour beaucoup de l'intérêt accordé à la perception subjective du temps dans la Recherche et de la posture essentiellement rétrospective du narrateur sur

\footnotetext{
${ }^{13}$ Ce que Bremond affirme d'ailleurs dans Logique du récit : " toute espèce de message narratif, quel que soit le procédé d'expression qu'il emploie, relève de la même approche à ce même niveau. Il faut et il suffit qu'il raconte une histoire. La structure de celle-ci est indépendante des techniques qui la prennent en charge. » (p. 11-12)
} 
cette question. Donc, voyons comment les quatre adaptations réagissent devant le problème de la temporalité proustienne.

Comme nous l'avons déjà souligné, Visconti veut demeurer fidèle au sentiment proustien, non au style. Cette philosophie se remarque dans le traitement temporel de son scénario. En fait, il n'est pas exagéré d'avancer que son scénario capitule devant la difficulté de créer un équivalent cinématographique de la temporalité proustienne, car globalement, le récit filmique de Visconti est très linéaire. Il ne contient qu'une infime quantité d'éléments inusités quant à la temporalité. Par exemple, un truchement visuel est utilisé lors d'une scène du deuxième voyage à Balbec. Il s'agit du moment où Marcel réalise la perte définitive de sa grand-mère. Visconti exprime le motif lié à la douleur de Marcel par un détail purement visuel, qui parvient à faire coexister le passé dans l'image du présent :

Debout, devant l'armoire, tournant le dos à la porte, une femme habillée de noir, la Grand'mère. C'est du moins ce que l'on pense un instant. Puis la femme se retourne vers Marcel - qui s'est assis dans le même fauteuil où nous l'avons vu une fois déjà, quand sa Grand'mère se préparait pour se faire photographier par Saint-Loup - et nous voyons que ce n'est pas la Grand'mère mais bien la mère de Marcel. (p. 73)

Ce trompe-l'œil, donnant l'impression que la grand-mère est présente dans l'image, crée un lien significatif entre le présent de Marcel et son souvenir du passé. Le procédé est d'autant plus satisfaisant qu'il est véritablement tiré du texte proustien, la mère de Marcel se substituant graduellement à l'image de la grand-mère, une fois cette dernière décédée. Visconti a donc récupéré ce motif du roman afin de représenter cinématographiquement le sentiment du narrateur prenant 
pleinement conscience du manque causé par la perte de sa grand-mère. Par ailleurs, le scénario de Visconti a d'intéressant temporellement qu'il termine son récit avec le début de la Recherche. En effet, le scénario conclut sur un plan de Marcel dans son lit, ayant commencé d'écrire des manuscrits, et visiblement très fatigué, ce qui rappelle le narrateur dormeur du début de la Recherche. Puis le scénario propose une métaphore visuelle liant le début à la fin de la Recherche : «Les feuilles manuscrites [...] se meuvent jusqu'à devenir semblables aux feuilles des arbres tranchant sur un ciel clair » (p.175). Ces feuilles d'arbres font bien évidemment référence à ceux de Combray. Cette métaphore visuelle entre les feuilles des manuscrits et les feuilles des arbres de Combray crée une boucle temporelle entre la fin et le début de la Recherche, imitant ainsi la circularité du texte proustien. Les dernières scènes du scénario de Visconti représentent Marcel enfant attendant anxieusement le son de la clochette lui indiquant que sa mère montera bientôt l'embrasser ${ }^{14}$. Sur le son de la clochette, les premiers mots de la Recherche sont murmurés par

\footnotetext{
${ }^{14}$ Le téléfilm de Companéez termine sur une note similaire, ce qui renforce l'hypothèse qu'elle se serait inspirée du scénario de Visconti. Le récit se termine alors que le narrateur est à l'Hôtel du prince de Guermantes se remémorant sa jeunesse, alors qu'il attendait avec impatience que M. Swann parte pour entendre le son de la clochette annonçant que sa mère allait monter à sa chambre pour l'embrasser. Le film se termine sur cette scène, alors qu'un gros plan montre les ardents baisers que le jeune Marcel prodigue à sa mère et que la voix over prononce ces mots: "il était temps de commencer». Nous ne pouvons nous empêcher de sourire quelque peu devant cette finale, qui place l'amour du jeune Marcel pour sa mère aux fondements mêmes de la nécessité d'écrire. Certes, l'amour obsessif du jeune Marcel pour sa mère est un élément important dans la révélation de la vocation littéraire, mais terminer le récit ainsi constitue une interprétation maternaliste très affirmée. Néanmoins, cette finale est plutôt efficace, car elle permet d'inclure en dernier lieu Du côté de chez Swann à l'œuvre, tout en recréant la circularité du texte proustien.
} 
le Marcel écrivain. Pour conclure sur la question de la temporalité du scénario de Visconti, il faut également souligner qu'au-delà des détails explicites contenus dans son texte, le réalisateur italien aurait œuvré à faire ressentir, cinématographiquement, le temps passé par cette esthétique de la nostalgie qui lui est si particulière. D'ailleurs, Henry Bacon propose une idée semblable à propos du projet proustien de Visconti : "The script would have enabled Visconti once again to recreate a bygone era; to dramatize people's fixity in time, place, class, historical and social situation; and show them in the penetrating yet tender light of critical nostalgia » (p. 210).

De son côté, Harold Pinter montre une plus grande attention que Visconti à créer des équivalences cinématographiques de la temporalité du texte proustien dans son scénario. À cet effet, il ne séquence pas le récit proustien d'après l'ordre narratif trouvé dans le roman. Il explique son choix ainsi :

Nous décidâmes de fonder l'architecture du film sur deux principes directeurs et contrastés: un mouvement, essentiellement narratif, vers la désillusion, et un autre, plus intermittent, vers la révélation, s'élevant vers le point où le temps perdu est retrouvé et se fixe à jamais en art. (p. 12)

Ce choix d'articuler narrativement son scénario selon ce double principe temporel est très ingénieux, car il permet à Pinter de présenter chronologiquement les scènes plus représentables cinématographiquement de la Recherche tout en décalant sans cesse la temporalité de son scénario par l'intrusion de fragments, visuels et auditifs, constituant des motifs révélateurs sur la future vocation littéraire de Marcel. Ces motifs sont 
presque tous introduits dès le début du scénario, durant les quelques premiers plans :

1. Écran jaune. Tintement d'une clochette de grille de jardin.

2. Rase campagne, une rangée d'arbres, vue d'un wagon de train arrêté. Aucun son. Fondu rapide.

3. Bref écran jaune.

4. La mer, vue d'une haute fenêtre, avec en premier plan une serviette accrochée à un porte-serviettes. Aucun son. Fondu rapide.

5. Bref écran jaune.

6. Venise. La fenêtre d'un palais, vue d'une gondole. Aucun son. Fondu rapide.

7. Bref écran jaune ${ }^{15}$.

[...]

26. Trois clochers d'églises, vus d'une calèche en mouvement, au crépuscule. Ils paraissent danser dans les derniers rayons du soleil.

27. Trois arbres, vus d'une calèche en mouvement, à midi. La calèche s'éloigne d'eux, et on a l'impression qu'ils la suivent. (p. 15-18)

On remarquera que la plupart de ces brefs motifs font tous référence à des souvenirs du narrateur, qui prendront tout leur sens lors de la révélation finale dans le petit salon du prince de Guermantes. Introduits dès le début du scénario, ils se représenteront de façon intermittente tout au long de celui-ci, comme les résidus d'un temps qui transcende la linéarité narrative. C'est par leur présence intrusive et itérative que Pinter souligne l'importance de ces moments qui, dans un élan commun, mèneront le narrateur à la création. Notons qu'ils servent également à reproduire cinématographiquement la circularité de l'œuvre proustienne, puisqu'on retrouve en

15 Il est à noter que ce «bref écran jaune » est en fait le petit pan de mur jaune de la Vue de Delft de Vermeer. 
conclusion du scénario un montage semblable à celui l'introduisant. Cette circularité est aussi redoublée par quelques plans présentés durant le montage introductif, qui montrent le Marcel du Temps retrouvé durant l'épisode du «Bal de têtes ». Ainsi, Pinter signifie d'emblée que ce qui suivra est déjà sous l'effet d'une remémoration et, comme le soulève Mark Graham, «because these images necessarily exist within the filmic present while referring implicitly to the narrative past, they admirably render the dual pastness and presentness of Marcel's memories » (p. 48). Par ailleurs, le script de Pinter exhibe d'autres formes de décalage temporel qui s'inscrivent comme des équivalences de la temporalité proustienne. Par exemple, à plusieurs reprises dans le scénario, on retrouve une série d'images rassemblées selon un principe analogique, images se référant à des moments temporellement éloignés les uns des autres. En fait, par ce procédé de montage par correspondance, Pinter cherche à créer un équivalent cinématographique de la narration proustienne et de son rapport à la mémoire. Prenons pour exemple le passage où, Albertine étant morte, le narrateur se questionne sur le malheur de sa situation amoureuse. À cet effet, il réfléchira à ses anciens amours, qui n'éveillent plus en lui la même passion, et il sera gravement attristé par l'anticipation que son amour présent pour Albertine s'estompera lui aussi avec le temps. Afin d'exprimer cette réflexion du narrateur cinématographiquement, Pinter propose une série de plans d'yeux des anciens amours de Marcel :

345. Yeux de Marcel.

346. Les yeux de Gilberte à Tansonville.

347. Les yeux de la duchesse de Guermantes dans la rue.

348. Les yeux d'Odette dans l'avenue des Acacias.

349. Les yeux de la mère dans la chambre de Combray. 
350. Les yeux de Marcel dans les cabinets de Combray.

351. Les yeux de Marcel. (p. 180)

Si ce montage ne restitue pas entièrement la réflexion du narrateur, le principe analogique demeure le même et se présente comme le produit, visuel, de la mémoire du personnage. Donc, nous avons constaté que le scénario de Pinter tente de restituer la temporalité proustienne à l'aide d'une structure temporelle où s'intercalent les motifs de la vocation littéraire ainsi que des montages de type mémoriel liant différents épisodes de la Recherche par analogie. De plus, le scénario souligne la circularité de l'œuvre par ces mêmes montages et par l'ajout d'une prolepse du Temps retrouvé placée au tout début du récit.

En ce qui concerne Un amour de Swann de Schlöndorff, on doit ici encore admettre que ce film prend une position peu audacieuse quant à communiquer la temporalité proustienne au cinéma. S'étant déjà émancipé du reste de l'œuvre en isolant la partie d'Un amour de Swann, la question de la temporalité proustienne ne semble pas vraiment préoccuper Schlöndorff. En fait, il va jusqu'à dissoudre le temps du récit en une seule journée, procédant à une condensation chronologique des événements narrés qui, dans la Recherche, s'étalent sur plusieurs années. On y perd évidemment toute l'importance accordée à l'écoulement du temps dans le roman, qui se fond désormais dans une suite contiguë d'actions dramatiques se chevauchant l'une après l'autre. On remarque toutefois une tentative d'actualiser le passé amoureux sous la forme d'une mémoire obsédante, vécue subjectivement par Swann. Le déclenchement mémoriel est d'ordre dramatique, puisqu'il vise essentiellement à mettre en contraste les temps heureux de sa 
relation avec Odette et sa présente jalousie maladive. Par exemple, Swann se remémore furtivement l'épisode des cattleyas à deux reprises durant le film, où le montage alterne entre son visage expressif (remémoration) et son souvenir. Un autre épisode profite du même traitement et survient lors d'une sieste du protagoniste, alors qu'il se rappelle, en songe, la première visite d'Odette à son appartement. Du moins, malgré le peu d'intérêt que Schlöndorff consacre à la temporalité proustienne, il prend la peine d'inclure un épilogue se passant sept années plus tard, narrant la visite de Swann chez la duchesse de Guermantes que l'on retrouve dans Le Côté de Guermantes. Swann annonce à la duchesse qu'il mourra d'ici quelques mois. Schlöndorff ajoute également un épilogue montrant une discussion entre Swann et Charlus sur la vie et la mort. La discussion s'interrompt lorsqu'Odette passe devant eux en carrosse. Le film se clôt sur Odette se promenant devant l'Arc de triomphe. Cette finale pose un terme définitif au récit, sans la moindre intention de suggérer la circularité narrative de la Recherche. D'ailleurs, le film se termine sur un arrêt sur image, soulignant que le récit est clos temporellement.

Le film de Ruiz, tout comme le scénario d'Harold Pinter, s'évertue particulièrement à transcrire cinématographiquement la temporalité proustienne. Contrairement à Pinter, qui décale la chronologie des événements, Ruiz profite plutôt de l'aspect récapitulatif du tome du Temps retrouvé pour créer des brèches temporelles avec les autres tomes de la Recherche. Le cinéaste chilien recrée cinématographiquement le principe proustien de la mémoire involontaire, en présentant plusieurs scènes de remémoration déclenchées par un stimulus sensible, dont la fameuse madeleine trempée dans le thé est demeurée l'exemple canonique (scène que Ruiz ne présente 
pas $\left.{ }^{16}\right)$. L'effet est très réussi cinématographiquement, puisque le décalage de l'image est immédiat, ce qui parvient à faire ressentir le phénomène au spectateur. Ce procédé sera particulièrement mis à profit lors de la dernière visite chez le prince de Guermantes, vu l'accumulation de ces incidents provoquant la mémoire involontaire chez Marcel, soit le pavé mal équarri, la serviette amidonnée, le bruit de la cuillère contre la tasse de thé, et le François le Champi de son enfance. Mais Ruiz multiplie ce principe de remémoration dans son film, et ce, afin d'y inclure des scènes appartenant aux autres tomes de la Recherche. D’ailleurs, ces scènes sont toutes suggérées dans Le Temps retrouvé. Ruiz n'opère donc pas un collage arbitraire, mais saisit plutôt ces opportunités afin de représenter des scènes appartenant aux volumes antérieurs, créant de la sorte des ponts temporels entre Le Temps retrouvé et le reste de la Recherche. Par exemple, durant le «Bal de têtes ", le narrateur fait cette réflexion en observant Odette :

Certes, Mme de Forcheville était depuis longtemps devenue une femme du monde. Mais recommençant à être entretenue sur le tard, et par un si orgueilleux vieillard qui était tout de même chez elle le personnage important, elle se diminuait à chercher seulement à avoir les peignoirs qui lui plussent, la cuisine qu'il aimait, à flatter ses amis en leur disant qu'elle lui avait parlé d'eux, comme elle disait à mon grand-oncle qu'elle avait parlé de lui au grand-duc qui lui envoyait des cigarettes; en un mot elle tendait, malgré l'acquis de sa situation mondaine, et par la force de circonstances nouvelles, à redevenir, telle qu'elle était apparue à mon enfance, la dame en rose. (p. 321)

\footnotetext{
16 Seule Companéez a « osé » filmer la scène de la madeleine trempée dans le thé, et il faut préciser qu'elle est présentée en guise d'introduction au deuxième volet du téléfilm (il fut diffusé en deux parties). Ainsi, la scène de la madeleine demeure en marge du récit à proprement dit.
} 
Ruiz profite de cette réflexion du narrateur rapprochant l'Odette du Temps retrouvé à celle Du côté de chez Swann pour présenter la scène entre le jeune Marcel, son grand-oncle Adolphe et la dame en rose telle qu'elle est décrite dans le Côté de chez Swann. Ainsi, plutôt que de verbaliser (en voix over vraisemblablement) la réflexion du narrateur, il insère une analepse représentant le souvenir de ce dernier. Par ce procédé, Ruiz résout en quelque sorte le problème du temps de l'image, car il montre ces analepses comme si elles prenaient place dans le présent de l'image, c'est-à-dire comme si elles apparaissaient telles quelles dans l'imagination du narrateur sous l'effet d'une remémoration involontaire. Le réalisateur met ainsi à profit le langage cinématographique dans son adaptation de la Recherche en créant ces brèches temporelles, qui sont ellesmêmes suggérées dans l'œuvre littéraire. De plus, tout en sachant que le lecteur proustien saura saisir le principe régissant l'insertion de ses scènes tirées des autres volumes du roman, en y prenant un certain plaisir d'érudition.

Cela dit, Ruiz est peut-être davantage ingénieux dans son traitement de la circularité de l'œuvre proustienne. Il parvient à émuler cette circularité narrative dans son film par quelques inventions de son cru figurant au début et à la fin du récit. L'affaire débute avec le prologue qui, comme nous l'avons dit, présente l'auteur au bord de la mort. Débuter avec cette scène à saveur biographique sert à mettre sur table la circularité de l'œuvre proustienne, car on entame le récit par la fin de la vie de l'auteur qui, on le sait, coïncide avec la fin de l'écriture de la Recherche. Le brio de Ruiz est d'avoir fictionnalisé ce fait lié à la vie de Proust, car cela redouble le principe circulaire de la Recherche voulant que nous lisions ce livre que le narrateur commencera à écrire à la fin du roman (ce qui n'est pas moins 
fictionnel). Ensuite, Ruiz crée une scène où sont entrecroisés certains éléments de la première partie Du côté de chez Swann ("Combray») et de la deuxième partie du Temps retrouvé, soient le début et la fin de la Recherche. Cette scène syncrétise le moment où le jeune Marcel joue avec sa lanterne magique dans sa chambre de Combray et ce passage du Temps retrouvé à propos des vieillards du «Bal de têtes » :

Des poupées, mais que pour les identifier à celui qu'on avait connu, il fallait lire sur plusieurs plans à la fois, situés derrière elles et qui leur donnaient de la profondeur et forçaient à faire un travail d'esprit quand on avait devant soi ces vieillards fantoches, car on était obligé de les regarder en même temps qu'avec les yeux avec la mémoire, des poupées baignant dans les couleurs immatérielles des années, des poupées extériorisant le Temps, le Temps qui d'habitude n'est pas visible, pour le devenir cherche des corps et, partout où il les rencontre, s'en empare pour montrer sur eux sa lanterne magique. Aussi immatériel que jadis Golo sur le bouton de porte de ma chambre de Combray. (p. 231, nous soulignons)

Dans le film de Ruiz, Odette demande aux invités du «Bal de têtes» de la suivre pour leur montrer quelque chose. Ce qu'elle désire leur montrer est en fait le jeune Marcel dans sa chambre de Combray jouant avec sa lanterne magique. Les invités se changent alors en statues ${ }^{17}$ et le jeune Marcel projette

17 Les multiples statues présentées dans le film de Ruiz constituent un motif visuel très important. Nous croyons que ces statues représentent deux concepts importants de la Recherche. D'une part, elle symbolise l'idée de l'écoulement du temps. Les statues sont des œuvres d'art sur lesquels le passage du temps est particulièrement perceptible, car elles sont susceptibles de changer d'apparence au fil du temps (s'effriter, se casser, changer de couleurs, etc.). En même temps, une statue représente également un moment fixe, le sujet étant figé dans une posture, elles préservent le corps en un instant bien précis. Ruiz s'intéresse probablement à ce paradoxe de la statue, qui englobe une thématique importante de l'œuvre proustienne, mais 
les images de sa lanterne magique sur ceux-ci. Cette scène brouille ainsi les limites temporelles entre ces deux passages très éloignés dans la Recherche, soulignant d'emblée la circularité de l'œuvre adaptée. Mais Ruiz ne s'arrête pas là. Suit alors la scène où le jeune Marcel débouche dans la salle aux chapeaux haut-de-forme, dans laquelle il voit Robert de SaintLoup (autre déroutement temporel). Marcel rejoint Robert en suivant un chemin précis à travers les chapeaux. Or, il y a une autre scène prenant place dans cette salle, qui survient alors que le narrateur quitte ses convives au salon du prince de Guermantes. Marcel prend une porte et se retrouve dans la salle aux chapeaux haut-de-forme ${ }^{18}$. Il reprend le même chemin qu'il

également parce que ce paradoxe temporel s'applique (quoique différemment) au médium cinématographique qui capture le mouvement de l'image, et donc l'écoulement du temps. D'autre part, en tant que motif persistant (voire obsédant) de son film et même de sa cinématographie (car les films de Ruiz en général regorgent de statues), elles sont un équivalent filmique de la petite phrase de Vinteuil, ce que l'analepse présentant Marcel et Albertine explicite. Alors que ce dernier explique la fonction artistique des phrases musicales qui se répètent dans l'œuvre de Vinteuil, la caméra effectue un travelling sur une série de statues placées en avant-plan, révélant en contrepoint avec le discours du personnage que les statues sont comme la petite phrase de Ruiz.

18 C'est à ce moment qu'on peut particulièrement mieux comprendre la signification de cette salle mystérieuse. Les chapeaux haut-de-forme, ainsi que les gants blancs disposés sur ces derniers, sont des signes très marqués de la mondanité. En ce sens, on peut interpréter que ces scènes signifient respectivement l'entrée et le départ du narrateur de la mondanité parisienne. D'ailleurs, dans le roman, le narrateur quitte pour de bon la société mondaine après sa visite chez le prince de Guermantes, et c'est bien cela qui est présenté dans le film lorsque Marcel prend cette porte qui le mène directement du salon du prince de Guermantes à la salle aux chapeaux haut-de-forme. Puisque le narrateur n'entame son œuvre qu'une fois qu'il a renoncé aux mondanités, on pourrait également avancer que cette salle représente une espèce d'antichambre de la création, la vocation littéraire du narrateur s'ancrant dans l'enfance (première scène) et se réalisant une fois sortie de la vie mondaine (deuxième scène). 
avait pris à travers les chapeaux pour se rendre auprès de Robert, mais cette fois, c'est un domestique qui l'attend. La scène suivante est également une invention de Ruiz, qui mélange des éléments de "Combray» et de la fin du Temps retrouvé. Elle présente l'épisode où le jeune Marcel entend le son de la clochette du jardin lui indiquant que sa mère viendra l'embrasser pour la nuit. Cependant, Ruiz y intègre un dialogue entre le jeune Marcel et le Marcel auteur qui reprend en fait des propos contenus dans les dix dernières pages du Temps retrouvé. Finalement, l'épilogue boucle la boucle avec le prologue, à l'aide d'une autre invention de Ruiz, soit la récitation ${ }^{19}$ d'une petite histoire à propos d'un sculpteur nommé Salvini. Sur le point de mourir, l'ange de la mort lui aurait apparu pour lui donner le temps de reparcourir sa vie avant son trépas. Néanmoins, le sculpteur voudrait plutôt prendre ce temps pour reparcourir sa dernière œuvre d'art. Cette histoire rappelle le prologue, qui nous présente un Marcel auteur au bord de la mort et nous invite à penser que le reste du film serait un peu comme une revisitation de son œuvre,

\footnotetext{
19 À première vue, on croirait que cette petite histoire est racontée en voix over, mais on peut voir dans l'image que c'est une femme, tenant un livre, qui la récite. L'histoire est donc narrée intradiégétiquement. En fait, l'utilisation de la voix over dans le film de Ruiz est judicieusement limitée. Alors qu'on pourrait s'attendre à une abondance d'interventions en voix over comme équivalence du discours littéraire, Ruiz a plutôt décidé d'utiliser cet effet à une seule cause et d'ainsi concentrer l'efficacité du procédé. Dans le film de Ruiz, la voix over sert uniquement à communiquer les pensées du personnage-narrateur en ce qui a trait à la révélation de sa vocation littéraire. Ainsi, elle sera utilisée pour exprimer ses doutes sur la littérature à partir de la lecture du journal des Goncourt, puis au moment où la révélation se manifeste alors qu'il est chez le prince de Guermantes.
} 
avant de mourir ${ }^{20}$. Ainsi, Ruiz parvient à communiquer cinématographiquement la circularité de la Recherche à partir des scènes ouvrant et clôturant le film, autant par le croisement d'éléments narratifs figurant au début et à la fin de la Recherche que par les nombreux parallélismes existant entre ces scènes. Le début du film préfigure la fin du récit et la fin du film rappelle le commencement du récit.

\section{La narration proustienne transposée au cinéma}

La dernière contrainte que nous analyserons, probablement le plus grand obstacle à toute adaptation cinématographique de la Recherche, est la transposition filmique de la narration proustienne. En effet, la littérarité proustienne est très difficilement transposable cinématographiquement puisque l'acte de narration littéraire lui est fondamental. Le narrateur proustien n'est pas une instance que l'on peut mettre de côté sans complètement défigurer l'essence esthétique de la Recherche. La subjectivité narrative est au cœur du texte proustien, et si utiliser la première personne du singulier dans un roman est une chose aisée, l'image, elle, ne dit jamais (ou difficilement) «je». De plus, dans le texte proustien, la subjectivité du narrateur s'exprime fortement par la constante présence d'un discours intériorisé à partir duquel tous les événements narrés sont canalisés ${ }^{21}$. Dans son article «One

20 Il s'agit également d'une analogie avec l'adaptation cinématographique d'œuvres littéraires qui est toujours une forme de revisitation de l'œuvre adaptée.

21 Le téléfilm de Companéez a beaucoup misé sur les ressources de la voix over pour préserver ce discours intériorisé. Bien que le film se borne surtout à mettre en scène les passages aisément représentables cinématographiquement de la Recherche, le flux narratif est continuellement ponctué de 
novel, five adaptations: Proust on film », Pascal A. Ifri discute des difficultés d'adaptation de la Recherche et soulève l'opposition entre une narration littéraire dite "fonctionnelle » et une autre, dite indicielle ${ }^{22}$. La narration fonctionnelle, c'est lorsque le point de vue narratif sert essentiellement à raconter des événements vus de l'extérieur. La narration indicielle, qui collerait davantage au texte proustien, c'est lorsque l'essentiel de la narration est centré sur le discours, et non sur les événements narrés pour eux-mêmes. Ifri en vient à ces conclusions :

The word "adaptation » would apply more to the filming of narratives that could not be easily distributed into segments because of their « heavily indicial » nature. Indeed, transferring to the screen "indices», the miscellaneous information regarding the characters and the general context, constitutes a much more complex task as it often requires radically transforming the material, such as using concrete elements for abstract ones or turning psychological data into images and sounds. Indeed, cinema does not have the flexibility of

pensées du narrateur récitées en voix over. Cette dynamique constitue un compromis bien mince quand on considère l'ampleur que le discours intériorisé occupe dans le roman, mais du moins, c'est une transposition qui a le mérite de fonctionner dans l'esthétique télévisuelle qu'affiche l'œuvre de Companéez. Néanmoins, on ne peut se cacher que le procédé est hautement conventionnel et souligne un manque d'originalité dans la réalisation de ce téléfilm.

22 Ces notions sont en fait dérivées de Roland Barthes et de son article « Introduction à l'analyse structurale des récits » (1966), où il propose que les deux grandes classes d'unités narratives soient les «Fonctions» et les "Indices », qu'il décline également en sous-catégories. Barthes propose même qu'une classification des récits puisse s'esquisser à partir de ces notions : « Ces deux grandes classes d'unités, Fonctions et Indices, devraient permettre déjà un certain classement des récits. Certains récits sont fortement fonctionnels (tels les contes populaires), et à l'opposé certains autres sont fortement indiciels (tels les romans "psychologiques"); entre ces deux pôles, toute une série de formes intermédiaires, tributaires de l'histoire, de la société, des genres. » (p. 9) 
literature and cannot easily switch from story to discourse or from the physical world to the mental one. Nor can it readily change tenses or points of view, or even, to quote [Seymour] Chatman again, «describe in the strict sense of the word, that is, arrest the action » (p. 16).

Donc, transposer la Recherche au cinéma demande un grand effort d'adaptation, puisque le texte proustien est davantage indiciel que fonctionnel, d'autant plus que la narration est fortement marquée par la subjectivité du narrateur, celui-ci passant sans cesse de la description du monde extérieur à sa pensée intérieure, la perception du premier toujours sculptée par l'analyse de cette dernière. Toutefois, il faut admettre que toutes les adaptations de la Recherche ont pu contourner ce problème lié à la narration essentiellement indicielle du roman, et ce, principalement pour deux raisons. La première, ironiquement, c'est l'immensité de l'œuvre, car même si la narration proustienne est plus indicielle que fonctionnelle, le roman contient assez d'éléments fonctionnels (ou événements narratifs) pour construire un scénario à partir des scènes représentables. D'ailleurs, les quelque 190 pages d'Un amour de Swann ont été suffisantes pour que Schlöndorff réalise un film d'un peu moins de deux heures. Dans toutes les adaptations, ce sont généralement les scènes représentables qui sont adaptées à l'écran (ou à l'écrit dans le cas des scénarios) et on peut remarquer comment la sélection et le traitement de ces scènes sont parfois quasi identiques d'un film à l'autre. Par exemple, aucune adaptation ne manque de présenter la scène dans l'Hôtel tenu par Jupien du Temps retrouvé, c'est-à-dire la révélation du penchant 
sadomasochiste de Charlus $^{23}$. Également, les scénarios de Pinter et Visconti adaptent la première soirée de Marcel chez la duchesse de Guermantes suivie de la désagréable visite chez le baron de Charlus. Cependant, si, dans le roman, la soirée chez la duchesse s'étale sur environ 130 pages et la visite chez le baron sur seulement une quinzaine de pages, la représentation de la soirée chez la duchesse est beaucoup moins développée dans les deux scénarios que la scène chez Charlus ${ }^{24}$. Ce débalancement entre le roman et les scénarios est dû aux différences dans la narration de ces passages dans l'œuvre littéraire. La narration de la soirée chez la duchesse est plus imbriquée dans la discursivité du narrateur, c'est-à-dire qu'il ne décrit pas l'événement dans sa contiguïté spatio-temporelle. Il rapporte ce qui s'est passé en sommaires plutôt qu'en dialogues ou encore en descriptions factuelles du déroulement, ce qui est difficile à segmenter et agencer pour former une scène représentable contiguë. C'est tout le contraire avec la visite chez M. de Charlus, où le dialogue et l'action sont prédominants et rapportés par le narrateur dans leur contiguïté spatiotemporelle. Les adaptations vont donc prioriser les moments de l'œuvre littéraire qui sont narrés comme cette visite chez le baron, où la narration est davantage «fonctionnelle». La

\footnotetext{
${ }^{23}$ Hormis le film de Schlöndorff centré sur Un amour de Swann. Évidemment, le téléfilm de Companéez représente la scène au bordel.

${ }^{24}$ Le téléfilm de Companéez représente également ces deux scènes contiguës et, contrairement aux scénarios de Pinter et Visconti, la première soirée chez la duchesse de Guermantes est plus longue que la visite chez le baron. Évidemment, elle est plus longue de quelques minutes, ce qui n'est pas équivalent à l'écart en pages du roman, mais l'effort de Companéez demeure intéressant. En fait, c'est surtout la dynamique entre la mise en scène et la voix over qui lui permet de développer davantage la scène chez la duchesse, ce qui imite partiellement la discursivité que l'on trouve dans ce passage du roman, à une échelle miniature toutefois.
} 
deuxième raison expliquant comment les adaptations peuvent contourner le problème de la narration proustienne, c'est qu'il est possible de regrouper en une séquence filmique des éléments qui sont dispersés dans le roman, et ce, qu'importe la nature indicielle ou fonctionnelle de la narration. Cette opération permet de pallier grandement la narration hautement indicielle de la Recherche, qui est plus perméable à la représentation cinématographique. Ainsi, les films peuvent demeurer fidèles à l'œuvre littéraire en rassemblant des éléments épars du roman tout en adaptant plus spécifiquement cette matière narrative au langage filmique. Comme le dit Mark Graham, "[this] compression would be necessary in any cinematic rendering of Proust» (p.45). Toutefois, coller ensemble des passages et des phrases dispersés dans la Recherche n'est pas garant d'une quelconque qualité d'adaptation de l'œuvre proustienne. Schlöndorff et Visconti sont ceux qui abusent le plus de ce procédé à des fins de compression et de dramatisation. Plutôt que de chercher des équivalences cinématographiques de la narration proustienne, ils préfèrent agencer le texte au profit d'une représentation dramatique (au sens théâtral) qui, du coup, gagne en autonomie par rapport à sa source littéraire. Car s'écarter du problème de la narration proustienne revient à se distancer de ce qui en fait une œuvre unique et remarquable. Certes, cela permet une plus grande flexibilité d'adaptation, mais le risque est de délaisser ce qui fait le génie de l'œuvre adaptée, soit l'origine même du désir de l'adapter au cinéma.

Cela dit, il est tout à fait compréhensible que les films contournent pour beaucoup le problème d'adapter cinématographiquement la narration proustienne, car à vrai dire, on ne peut décidément pas la transposer parfaitement au sein d'une 
œuvre filmique. Néanmoins, il existe un compromis pouvant résoudre partiellement l'impasse, compromis qui consiste à destiner l'adaptation à un groupe restreint de spectateurs, soit les lecteurs de Proust. C'est vraisemblablement ce que Ruiz et Pinter ont fait, et tous deux marquent ce parti pris au début de leur film/scénario. Nous avons déjà soulevé comment le scénario de Pinter propose en introduction une série d'images représentant des motifs importants de la Recherche. Or, ces motifs ne peuvent être reconnus que par un spectateur qui a déjà lu Proust, qui est donc apte à saisir qu'il s'agit de lieux et de moments associés à la révélation de la vocation littéraire du narrateur dans le roman. Chez Ruiz, on comprend, durant le prologue, que le film s'adresse à des lecteurs de Proust alors que l'auteur regarde des photographies et y reconnaît les personnages de la Recherche. Il ne s'agit pas d'une introduction aux personnages à proprement dit. Il ne donne aucune information sur ceux-ci, il ne fait qu'énoncer leur nom afin que le spectateur/lecteur puisse se remémorer le personnage proustien et l'identifier visuellement à l'acteur qui l'interprète. Le spectateur n'ayant aucune connaissance de la Recherche n'apprendra rien sur les personnages à ce moment, sauf leur nom. Donc, tenant pour acquis que leur adaptation s'adresse tout particulièrement aux spectateurs ayant une certaine connaissance de la Recherche, Pinter et Ruiz peuvent considérer que ceux-ci sauront reconnaître les équivalences cinématographiques de la narration proustienne suggérées dans leur film.

Le scénario d'Harold Pinter parvient à représenter la subjectivité du narrateur proustien, et ce, en identifiant l'image cinématographique à la mémoire de celui-ci. Nous utiliserons encore ici le montage du prologue pour exemplifier le procédé. 
Les 23 premiers plans du scénario alternent entre des motifs visuels, qui sont soit représentés sans son, soit accompagnés de motifs auditifs tels le son de la clochette du jardin et la musique de Vinteuil, ainsi que des plans montrant la visite de Marcel chez le prince de Guermantes dans la deuxième partie du Temps retrouvé, accompagnés de sons ambiants réalistes. Pinter suggère que les plans muets, ou ceux accompagnés de motifs auditifs précis (clochette, Vinteuil) sont des images mentales attribuables à la mémoire subjective du narrateur proustien. Il accentue cette marque subjective en plaçant le point de vue de la caméra d'après une perspective humaine: "une rangée d'arbres, vue d'un wagon de train arrêté [...] La mer, vue d'une haute fenêtre [...] Venise. La fenêtre d'un palais, vue d'une gondole» (p.15). Dans les plans réalistes (chez le prince de Guermantes), Marcel est dans l'image, au contraire des images mentales qui seraient perçues par son regard intérieur. Pinter ajoute une autre démarcation esthétique à ce montage en prévoyant filmer les scènes réalistes en noir et blanc et les images mentales en couleurs, donnant ainsi une plus forte résonance affective aux images mémorielles du narrateur. Mark Graham dit de ce montage qu'il «implies an appropriation (quasi-narratorial) of the ostensibly objective reality by Marcel's subjectivity» (p. 42). Ainsi, ce prologue place métaphoriquement le point de vue intérieur de Marcel comme la source de l'image cinématographique, suggérant que le film en entier peut s'interpréter comme une remémoration subjective de l'expérience de Marcel. Évidemment, il est plus aisé de saisir cette idée si on a préalablement lu la Recherche, car la signification des motifs visuels et auditifs présentés ne va pas de soi, tout comme le contexte des plans représentant la visite chez le prince de Guermantes. Seul un spectateur déjà 
initié à l'œuvre proustienne saura interpréter le prologue en ce sens.

Le film de Raoul Ruiz tente également de transposer cinématographiquement le point de vue narratif de la Recherche, mais il ne mise pas sur une équivalence filmique de la subjectivité du narrateur pour y arriver. Le Temps retrouvé de Ruiz est une lecture délibérément cinématographique de la Recherche, en ce sens qu'il ne cherche aucunement à recréer une quelconque forme d'intériorité mentale de l'image. À l'inverse, le film met à profit les spécificités expressives du cinéma en inscrivant le point de vue narratif dans l'extériorité de l'image. Jean Bessière discute du phénomène ainsi :

Le Temps retrouvé de Raoul Ruiz est doublement un film de l'extériorité : en tant qu'il est un film; en tant qu'il fait du narrateur de la Recherche, Marcel, le spectateur explicite de ce que ce narrateur est supposé avoir écrit et qui se présente à lui comme un spectacle qu'il visite, sous la forme de scènes réelles qui apparaissent sous la forme d'images sur l'écran. (p. 8)

En effet, Ruiz confine le personnage du narrateur dans l'univers profilmique, mais il hypertrophie son rôle d'observateur (qui n'est pas sans rapport avec la narration littéraire), au point d'en faire un spectateur intradiégétique. Le narrateur n'est pas la source de l'image diégétique, il est un observateur à l'intérieur de celle-ci. De plus, Ruiz souligne incessamment la présence du narrateur dans l'espace diégétique. Par exemple, à l'aide de brèves apparitions dans une scène où il n'est pas impliqué directement, comme c'est le cas durant le dialogue entre madame Verdurin et Morel à propos des croissants qui soulagent ses migraines, où la caméra opère un panoramique insoupçonné pour découvrir, dans le coin de la 
pièce, Marcel qui regardait la scène. Également, on remarque à quelques reprises le narrateur réfléchi dans un miroir ou le reflet d'une vitre. On affirme aussi sa présence dans l'image en ayant son ombre dans le champ. Plus étonnant encore, le narrateur fait partie des invités dans la scène représentant le pastiche des Goncourt. Or, ce choix révèle sans ambiguïté le projet de Ruiz, car, dans le roman, la lecture du journal des Goncourt provoque une réflexion chez le narrateur sur la représentation du réel par la littérature, justement car il est absent d'une narration dont il connaît réellement les «personnages", ayant dîné à plusieurs reprises chez les Verdurin. Inclure le narrateur dans la diégèse du journal des Goncourt constitue pour Ruiz un moyen de signaler le rôle de spectateur intradiégétique du narrateur, car il lui fait transgresser la vraisemblance diégétique. Qu'importe le niveau diégétique (le monde de la Recherche est métadiégétique par rapport au pastiche des Goncourt), la place du narrateur est celle d'observateur dans l'image, ce qui a pour effet de stratifier l'image en plusieurs niveaux diégétiques. Cette présence obsédante du narrateur dans l'image cinématographique incite à interpréter d'autres éléments du film comme découlant d'une adaptation filmique de la narration proustienne. D'ailleurs, Le Temps retrouvé est la seule adaptation d'après la Recherche qui parvient à transposer le ton ironique du narrateur romanesque. Ce ton ironique se traduit particulièrement par le jeu caricatural des personnages, affichant même des tics gestuels et verbaux qui les rendent passablement ridicules. En outre, l'interprétation du narrateur par Marcello Mazzarela accuse une touche burlesque qui n'est pas sans rappeler le personnage 
de Charlot ${ }^{25}$. Ces effets comiques de mise en scène constitueraient donc une forme d'adaptation du discours ironique de la narration proustienne. Un autre aspect stylistique de la narration proustienne trouvant un équivalent cinématographique dans Le Temps retrouvé est l'usage de l'analogie comparative (ou métaphorique). Ruiz traduit cinématographiquement ces figures de style par l'utilisation de surimpressions filées en fondu enchaîné. Ce procédé est un motif esthétique très marqué dans le film, Ruiz l'utilisant à plusieurs reprises ${ }^{26}$, dont le plus remarquable exemple est peut-être lorsque Gilberte s'habille comme Rachel. La juxtaposition des deux femmes par surimpression semble nous communiquer visuellement une loi proustienne sur l'amour, selon laquelle on retrouverait toujours un peu de nos anciens amours dans la personne qu'on aime (le sujet aimant ici étant Robert). Le film de Ruiz est également attentif au principe proustien voulant qu'une personne soit investie de plusieurs «moi » au cours de son existence. Ainsi, il y a quatre acteurs dans le film qui jouent le narrateur; l'auteur du prologue, le

${ }^{25}$ Ce que Jérôme Cornette remarque dans son article « Raoul Ruiz : une pensée-cinéma de Proust»(p. 205). Par ailleurs, Antoine Compagnon avait proposé une comparaison entre le narrateur et Charlot en discutant de la scène dans l'arrière-cour de l'Hôtel de Guermantes dans son livre Proust entre deux siècles (p. 13). Il n'est pas impossible que Ruiz ait appliqué le propos de Compagnon pour son film.

${ }^{26}$ Il semblerait que l'article «Le cinéma à la recherche du temps perdu » de Jacques Bourgeois serait à l'origine de cette surenchère de surimpressions filées en fondu enchaîné dans le film de Ruiz. Bourgeois propose dans ce texte que le style littéraire de Proust préfigure certaines possibilités langagières du cinéma, ce qu'il illustre à l'aide de comparaisons avec Citizen Kane d'Orson Welles (1941). Bourgeois soulève un rapprochement entre l'usage de la métaphore chez Proust et le procédé du fondu enchaîné, ce que Ruiz applique avec une telle insistance dans son film que l'on ne peut qu'en déduire qu'il s'est inspiré du texte de Bourgeois. 
jeune Marcel de Combray, l'adolescent à Balbec et le Marcel adulte. Ils servent bien sûr à représenter le personnage à différents âges de sa vie, mais Ruiz profite de ce qui pourrait se réduire à un souci de vraisemblance afin d'exprimer cinématographiquement la complexité du «je» proustien. L'auteur représente le « je » qui a écrit, ce qui est confirmé dès le prologue lorsque le personnage dicte la dernière page écrite par Proust; le Marcel adulte est ce narrateur observateur dont nous discutions plus haut; le jeune Marcel est identifié au signe de la mémoire, comme un lien intérieur entre la sensation et le souvenir, ce qui explique d'ailleurs pourquoi Ruiz représente Marcel à l'âge de l'enfance dans la remémoration du voyage à Venise, alors qu'il devrait vraisemblablement être d'âge adulte à ce moment. Ruiz fait interagir ensemble ces trois personnages du narrateur, ceux-ci se retrouvant parfois simultanément dans l'image et entretenant même un dialogue durant les derniers plans du film. Ce dispositif sert à extérioriser une donnée purement intérieure de la narration proustienne, soit le sentiment d'existence stratifié par la mémoire. L'expression littéraire peut aisément communiquer ce «dialogue » intérieur entre les différents «moi» d'une personne (le narrateur proustien). Le brio de Ruiz est d'avoir pensé une manière de représenter ce principe cinématographiquement, c'est-à-dire en le rendant visible tout en signifiant, par une mise en scène surréaliste, qu'il s'agit d'un phénomène intérieur. Le Temps retrouvé présente beaucoup d'autres procédés de la sorte qui rendent visibles certains principes de la narration proustienne. À bien des égards, seul un lecteur érudit de l'œuvre de Proust pourra les identifier, car une connaissance préalable du texte littéraire est requise pour saisir les équivalences cinématographiques proposées par Ruiz qui, nous devons le 
souligner, ne sont pas toujours aisées à comprendre, même pour un lecteur de Proust aguerri.

Ainsi donc, ces trois contraintes à l'adaptation cinématographique d'À la recherche du temps perdu de Marcel Proust nous ont permis de faire un parcours critique à travers quatre adaptations. De ce parcours, nous avons pu remarquer comment il devient problématique de réaliser une adaptation cinématographique de la Recherche voulant trop s'écarter de la littérarité de l'œuvre. Nier les difficultés intrinsèques à la transposition de l'essence proustienne au cinéma tend à produire des œuvres peu efficaces esthétiquement, qui se contentent d'un défilé de scènes représentables contenues dans le roman ${ }^{27}$. Ironiquement, procéder de la sorte répond à ce que le narrateur proustien dit lui-même à propos d'une certaine littérature de notations à éviter : "Quelques-uns voulaient que le roman fût une sorte de défilé cinématographique des choses. Cette conception était absurde. Rien ne s'éloigne plus de ce que nous avons perçu en réalité qu'une telle vue cinématographique. » (Proust, 1989, p. 189) En effet, il est regrettable de faire d'une œuvre littéraire aussi complexe que la Recherche un simple «défilé » de scènes cinématographiques conventionnelles. Comme l'indique David Davidson, «the screen version of Remembrance of Things Past demand a form as revolutionary as that of the novel » (p. 168). L'adaptateur doit s'évertuer à créer des équivalences filmiques de la temporalité et de la narration proustienne. À cet égard, il nous semble

27 Ce dont le téléfilm de Companéez souffre particulièrement. 
préférable de compter sur l'érudition du spectateur-lecteur de Proust, qui peut saisir les signes cinématographiques qui renvoient à l'esthétique proustienne. Le scénario de Pinter et surtout le film de Ruiz ont déjà ouvert la voie dans cette direction. Par ailleurs, cette solution semble se justifier d'ellemême, car à quoi bon adapter la Recherche si ce n'est pour y communiquer son ingéniosité artistique, qui est loin de se résumer à la lettre de son récit. Expliquer l'essence proustienne par un film en partant du point zéro est une entreprise inconcevable, alors qu'à l'inverse, s'appuyer sur le présupposé que le spectateur connaît la Recherche offre une possibilité infinie de dialogues sur l'œuvre adaptée. Espérons que l'avenir nous réserve d'autres audacieuses tentatives d'adaptation. Qui sait, peut-être que quelques audacieux entreprendront de réaliser les scénarios de Pinter et Visconti. Cela dit, la Recherche est une œuvre immense et riche et ces quatre adaptations n'ont qu'effleuré les possibilités qu'offre l'adaptation cinématographique de ce chef-d'œuvre littéraire. Même en termes de scènes représentables, les cinéastes sont loin d'avoir épuisé les ressources fournies par l'œuvre proustienne. Pensons comment la soirée chez la princesse de Guermantes, avec l'épisode de la fameuse fontaine, dont les jets d'eau sont équivalents au jeu des classes sociales, ferait une belle séquence filmique. Ou encore la visite dans l'atelier d'Elstir à Balbec ${ }^{28}$, qui invite à des délices

\footnotetext{
${ }^{28}$ Le téléfilm de Nina Companéez présente une scène dans l'atelier d'Elstir à Balbec, où nous pouvons apercevoir dans quelques plans des tableaux à saveur impressionniste. Cependant, il faut avouer que cette scène ne s'attarde pas vraiment à la réflexion sur l'art que les œuvres d'Elstir provoquent chez le narrateur dans le roman, ce qui est à notre avis l'élément le plus intéressant de la rencontre du narrateur avec le peintre. Dans le téléfilm, c'est surtout le point de rencontre entre le narrateur et les jeunes filles en fleur que constitue l'atelier d'Elstir qui est retenu.
} 
picturaux qui seraient de véritables défis pour l'image cinématographique. À noter que Vincent Descombes consacre un chapitre complet sur l'atelier d'Elstir dans son livre Proust. Philosophie du roman (p. 272-291) dans lequel il propose que le tableau Le Port de Carquehuit, tel que décrit dans la Recherche, soit tout simplement irreprésentable. Or, non seulement cette idée constitue une sorte de résumé de la difficulté d'adaptation cinématographique de l'œuvre proustienne, mais ci-gît également tout l'intérêt d'une telle entreprise. Car l'irreprésentabilité de la description littéraire du tableau d'Elstir s'ouvre proportionnellement aux possibles de l'interprétation cinématographique, sans quoi aucun acte d'adaptation ne serait véritablement créateur.

\section{Filmographie}

COMPANÉEZ, Nina. (2011), À la recherche du temps perdu, Ciné Mag Bodard avec la participation de France Télévisions, Arte France et TV5Monde, 220 minutes.

RUIZ, Raoul. (1999), Le Temps retrouvé, d'après l'œuvre de Marcel

Proust, Gemini Films, 158 minutes.

SCHLÖNDORFF, Volker. (1984), Un amour de Swann, Gaumont, 110 minutes.

STÉPHANE, Roger. (1962), Portrait-souvenir : Marcel Proust, INA, 87 minutes.

\section{Bibliographie}

BACON, Henry. (1998), Visconti: Explorations of Beauty and Decay, New York, Cambridge University Press. 
BARTHES, Roland. (1966), "Introduction à l'analyse structurale des récits », Communications, vol. 8, p. 1-27.

BESSIÈRE, Jean. (2001), « Deux fois le temps retrouvé : Raoul Ruiz et Proust. L'idéalisme de la littérature, l'idéalisme du cinéma et le temps de la visitation », Estudos Literários / Estudos Culturais, Actas do IV Congresso Internacional da Associação Portuguesa de Literatura Comparada, Associação Portuguesa de Literatura Comparada / Universidade de Évora, CD-ROM (2004), vol. III.

BLUESTONE, George. (1971 [1957]), Novels into Film, Berkeley, University of California Press.

BOURGEOIS, Jacques. (1946), « Le cinéma à la recherche du temps perdu », La Revue de cinéma, no 3, p. 18-37.

BRATER, Enoch. (1979), « Time and Memory in Pinter's Proust Screenplay », Comparative Drama, vol. 13, n 2, p. 121-126.

BREMOND, Claude. (1973), Logique du récit, Paris, Seuil.

CARRIER-LAFLEUR, Thomas. (à paraître), L'OEil cinématographique de Proust, Paris, Classiques Garnier, coll. "Bibliothèque proustienne ».

CECCHI D'AMICO, Suso et Luchino VISCONTI. (1984), À la recherche du temps perdu. Scénario d'après l'œuvre de Marcel Proust, Paris, Persona.

COMPAGnon, Antoine. (1989), Proust entre deux siècles, Paris, Seuil.

CORNETTE, Jérôme. (2001), « Raoul Ruiz : une pensée-cinéma de Proust », Revue Critique, no 646, p. 197-208.

DAVIDSON, David. (1982), « Pinter in No Man's Land: The Proust Screenplay», Comparative Literature, vol. $34, n^{\circ} 2$, p. 157170.

Descombes, Vincent. (1987), Proust. Philosophie du roman, Paris, Minuit, coll. «Critique». 
GRAHAM, Mark. (1982), «The Proust Screenplay: Temps Perdu for Harold Pinter? », Literature/Film Quarterly, vol. 10, n 1 , p. 38-52.

IFRI, Pascal A. (2005), « One Novel, Five Adaptations: Proust on Film », Contemporary French and Francophone Studies, vol. 9, no 1 , p. 15-29.

PINTER, Harold. (2003 [1978]), Le Scénario Proust: À la recherche $d u$ temps perdu, traduit par Jean Pavans, Paris, Gallimard, coll. « Du monde entier ».

PRoust, Marcel. (1930), Un amour de Swann, Paris, Gallimard.

—. (1989 [1927]), Le Temps retrouvé, Paris, Gallimard, coll. «Folio classique».

TRUFFAUT, François, Alfred HITCHCOCK \& Helen G. SCOTT. (1972 [1967]), Hitchcock/Truffaut (revised edition), New York, Simon \& Schuster.

\section{Résumé}

Cet article s'intéresse aux difficultés inhérentes à l'adaptation cinématographique d'À la recherche du temps perdu de Marcel Proust. Trois contraintes d'adaptation sont ciblées particulièrement, soit le problème de la longueur de la Recherche, la complexité de sa structure temporelle et la difficulté à transposer la narration proustienne à l'écran. L'auteur présente chacune des contraintes et offre une description des problèmes découlant de leur transfert du roman au médium cinématographique, pour ensuite analyser comment quatre tentatives d'adaptation ont tâché de résoudre ces contraintes. Les adaptations discutées sont les scénarios de Luchino Visconti (À la recherche du temps perdu) et d'Harold 
Pinter (Le Scénario Proust) ainsi que les films Un amour de Swann de Volker Schlöndorff et Le Temps retrouvé de Raoul Ruiz. La diversité des approches présentées par ces adaptations montrera l'intérêt et le défi artistique que constitue la transposition de la Recherche au cinéma.

\begin{abstract}
This article shows interest in some inherent difficulties to the film adaptation of Marcel Proust's In Search of Lost Time. Three adaptation constraints are particularly discussed: the problem of the lenght of Proust's work, the complexity of its temporal structure and the difficulty of transposing proustian narration to the screen. The author first introduces each of these contraints and offers a description of key problems arising from their transfer from the novel to the film medium, and then analyzes how four adaptation attempts tried to solve these constraints. The film adaptations discussed are scenarios by Luchino Visconti (In Search of Lost Time) and Harold Pinter (The Proust Screenplay), as well as films by Volker Schlöndorff (Swann in Love) and Raoul Ruiz (Time Regained). The diversity of approaches shown by these film adaptations will demonstrate the highly artistical challenge which constitutes translating Proust's novel into film.
\end{abstract}

